\title{
Metabolic syndrome and its individual components among people with type 2 diabetes: Prevalence, gender differences and its association with ischemic heart disease
}

\author{
Ali Khan Khuwaja ${ }^{1}$, Farzana Nawaz $\mathrm{Ali}^{2 \#}$, Imama Naqvi ${ }^{3}$, Rasool Bux ${ }^{4}$, Abdul Jabbar ${ }^{5}$, \\ Raheem Hassan Dhanani ${ }^{6}$ \\ ${ }^{1}$ Department of Family Medicine and Community Health Sciences, Aga Khan University, Karachi, Pakistan; \\ fn.khuwaja@yahoo.com \\ ${ }^{2}$ Department of Family Medicine, Aga Khan University, Karachi, Pakistan; ${ }^{\#}$ Corresponding Author: fn.khuwaja@yahoo.com \\ ${ }^{3}$ Division of Neurology, Department of Medicine, Aga Khan University, Karachi, Pakistan; imama.naqvi@gmail.com \\ ${ }^{4}$ Department of Community Health Sciences, Aga Khan University, Karachi, Pakistan; rasool.bux@aku.edu \\ ${ }^{5}$ Aga Khan University, Karachi, Pakistan; abdul.jabbar@aku.edu \\ ${ }^{6}$ Department of Family Medicine, Aga Khan University, Tanzania, East Africa; raheem.dhanani@aku.edu
}

Received 15 April 2012; revised 17 May 2012; accepted 29 May 2012

\section{ABSTRACT}

Background: Metabolic syndrome (MS) is common among people with type 2 diabetes. The purpose of this study was to assess the proportion of MS and its individual components among type 2 diabetic adults, to stratify these by gender and to determine their association with ischemic heart disease (IHD). Method: A cross-sectional multicentre study was conducted in public and private sector clinics in Karachi, Pakistan. A pretested structured questionnaire and medical records were used to collect information from 889 people with type 2 diabetes after informed consent. Chi-square test and odds ratios with $95 \%$ $\mathrm{Cl}$ were used to assess the association of MS, its components and IHD. Adjusted odds ratios were used to evaluate the differences of MS and its components by gender. Results: In all, $70 \%$ of the participants had MS. The likelihood of having IHD among those with MS was higher (OR = 3.21; $95 \% \mathrm{Cl}=2.22-4.65)$. Of the individual MS components, obesity conferred the strongest association with IHD $(\mathrm{OR}=2.57 ; 95 \% \mathrm{Cl}=1.91$ - 3.43). Multivariate analysis showed an increased proportion of MS among women (AOR = 1.63; 95\% $\mathrm{Cl}=1.18$ - 2.24). Of the individual MS components, women had a higher prevalence of obesity $(A O R=4.70 ; 95 \% \mathrm{Cl}=3.30-6.70)$. Conclu-

"Conflicts of interest: The authors have not declared any conflict of interest. sion: A very high burden of MS among type 2 diabetics has been identified in this study. Dedicated interventions are needed to prevent/ delay the onset of MS and the associated morbidity and mortality.

Keywords: Type 2 Diabetes; Metabolic Syndrome; Ischemic Heart Disease; Gender Differences;

Developing Country

\section{INTRODUCTION}

Diabetes Mellitus is being increasingly viewed as a rising pandemic worldwide, with developing countries bearing the major brunt of the disease. Estimated to have affected approximately $6.4 \%$ of the adult population in 2010 globally, the prevalence of diabetes is expected to rise to $7.7 \%$ by 2030 [1]. It is also projected that by the same year, the developed and the developing countries will see a $20 \%$ and $69 \%$ increase respectively, in the number of adults with diabetes [1]. When considering impaired glucose tolerance with diabetes, the prevalence of total glucose intolerance may even be higher, predicting an alarming burden of the disease in future [2].

Diabetes Mellitus leads to a greater risk for all manifestations of atherosclerosis, particularly coronary artery, cerebrovascular and peripheral arterial diseases $[3,4]$. These macrovascular complications cause significant morbidity and mortality, such that approximately, 80\% of the total mortality in diabetic patients is attributed to some major cardiovascular complication [4]. Among the chronic complications of diabetes, the management 
of cardiovascular disease (CVD) accounts for the major share of expenditure and resources used in the care of diabetics, posing substantial economic burden $[5,6]$. Ischemic Heart Disease (IHD) the most frequent single cause of death among diabetics [7] is known to be related to certain risk factors like hypertension (HTN), dyslipidemia, microalbuminuria, and obesity $[3,4,8]$. There is considerable evidence to suggest that modification of these factors results in a substantial decrease and delay in the development of heart diseases [8,9]. Metabolic syndrome (MS) is a clustering of such risk factors and its prevalence is found to be nearly $60 \%$ to $80 \%$ among diabetic patients in different parts of the world [10-13]. Although, MS is known as a significant and independent predictor of cardiovascular disease, its presence among persons with type 2 diabetes raises this risk to the greatest $[10,14,15]$.

Ischemic Heart Disease was largely considered a disease more prevalent among men for a long time. However recent evidence clearly points to the increased prevalence among women [16]. Although the degree of definite coronary artery disease may be twice as high among males, the electrocardiographic evidence of ischemia is seen more among females [16]. These findings can be well attributed to the much greater burden of CVD associated factors that have been observed to prevail and cluster among women [17-19] that may overwhelm the protective role of the female sex hormones against the development of atherosclerosis. Several studies on MS among people with type 2 diabetes have also clearly revealed an increased existence of MS components and their clustering among female gender [12,13]. However, there still exists a profound need to thoroughly assess the individual MS components with respect to gender in this population. The presence of diabetes, along with other MS components, makes it imperative to identify the differences in the metabolic risk profile between both the genders, so that in the setting of paucity of resources in developing countries, it becomes possible to plan and prioritize interventions for high risk groups. We therefore, in this study assessed the proportion of MS and its individual components and, estimated their association with IHD among people with type 2 diabetes visiting outpatient clinics in Karachi, Pakistan. We also identify the differences of MS and their individual components by gender.

\section{METHODS}

We conducted a cross-sectional multi-centre study in four out-patient clinics in Karachi, a cosmopolitan city and economic hub of Pakistan, with a population of over 16 million. In order to capture a diverse population with respect to socio-economic strata and clinical spectra, and to render the results more generalizable, both public and private sector clinics were approached. The four clinics included in the study represented family medicine, internal medicine, specific diabetes care and endocrine clinics.

Selection and Description of participants: We included in our study, all persons with an established diagnosis of type 2 diabetes attending clinics for follow-up visit. Excluded were people with type 1 diabetes, gestational diabetes and diabetes secondary to other diseases and/or drugs. Although no harm was expected to occur to any of the study participant, the study questionnaire was reviewed and approved by the Research Committee of the department of Family Medicine, Aga Khan University, Karachi. Permission was also taken from each of the studied clinic.

Informed consent was obtained from the participants after explaining the procedure and the objectives of the study, and complete confidentiality of the collected data was ensured to them.

All data were collected and edited by medical graduates specifically trained for this task. Of the 1000 eligible people with type 2 diabetes approached consecutively at the four clinics, 87 patients refused to participate while information was missing for 24 records. Hence, the final analysis was done on 889 participants.

Technical Information: A pretested structured questionnaire was used to collect data on the socio-demographic and clinical characteristics. Some variables were also confirmed from patients' medical records. For this study, physical activity was defined as "regular" if a person brisk walked or exercised for a minimum of 20 minutes, for four or more times per week. Smoking status of the participant was regarded as "current smoker" if the respondent was currently smoking and smoked at least 100 cigarettes in lifetime. Individuals were classified as hypertensive if they were previously diagnosed and were currently on anti-hypertensive medication. A history of IHD was considered to exist if a history of angina and/or myocardial infarction was elicited from the responders and/or documented in the medical records. Besides these, patients' medical records were used to obtain other variables such as height, weight, blood pressure (BP), fasting blood glucose (BG) and fasting blood triglycerides (BT). For this study, we used the definition of MS as suggested by World Health Organization [20] which is one of the most widely used criteria among people with diabetes.

Data were analyzed using SPSS-version 19. Proportions were calculated for all the variables of interest. Univariate analysis was carried out to assess the relationship between the outcome variables (MS and its individual components) and IHD by using chi-square test and crude odds ratio (OR) with 95\% Confidence Interval (CI). Univariate analysis was also done to evaluate the relation- 
ship between MS, its components and gender categories. Multiple logistic regression was done after adjusting for the confounding variables. Results are expressed as adjusted odds ratio (AOR) with 95\% CI. The components of MS (systolic BP, diastolic BP, fasting BG, fasting BT and $\mathrm{BMI}$ ) were taken as continuous variables in assessing the association with IHD and gender.

\section{RESULTS}

Respondents of our study had a predominance of females (57.5\%). More than half the subjects were 50 years of age and 59.5\% were found to have diabetes for 5 years. Almost two thirds of the participants were not physically active at the time. Socio-demographic characteristics are detailed in Table 1.

IHD was documented among 28\% (95\% CI: 25.5 31.2) of studied type 2 diabetic patients whereas MS was present in $71.7 \%$ (95\% CI: 68.9 - 74.6) of the subjects. Hypertension was the most common component of MS noted in 60.2\% (95\% CI: 57.1 - 63.3) patients.

Associations for MS and its individual components with IHD among people with type 2 diabetes are summarized in Table 2. MS and all its individual components were found to be significantly associated with IHD. However, the odds of having IHD among subjects with MS were substantially higher compared to individual components of MS. The likelihood of having IHD among subjects with MS was more than three times higher compared to those without MS (OR = 3.21; 95\% CI: 2.22 - 4.65). Of the individual components of MS, obesity conferred the strongest association with IHD. The odds of having obesity were more than two and a half times higher in persons with IHD (OR $=2.57$; 95\% CI: 1.91 3.43). The odds of the other components of MS such as HTN (OR = 1.39; 95\% CI: 1.10 - 1.90), microalbuminuria $(\mathrm{OR}=1.83 ; 95 \% \mathrm{CI}=1.36-2.45)$ and dyslipidemia $(\mathrm{OR}=1.84 ; 95 \% \mathrm{CI}=1.38-2.45)$ were also higher among persons with IHD.

Proportion of MS and its individual components stratified by gender among the study population are given in Table 3. Multivariate analysis done after adjusting for age, physical activity and smoking status revealed a higher MS prevalence among women as opposed to men (women: $73.8 \%$ vs men: $63.5 \%$; $\mathrm{AOR}=1.63$; $95 \% \mathrm{CI}=$ 1.18 - 2.24). Of the individual MS components, obesity prevalence was significantly higher among women compared to their counter parts $(\mathrm{AOR}=4.70 ; 95 \% \mathrm{CI}=3.30$ - 6.70). As opposed to that, microalbuminuria was seen less frequently among women than men $(\mathrm{AOR}=0.70$; $95 \%$ CI $=0.51$ - 0.95), same was also true for dyslipidemia. Although HTN was seen slightly more among women, however the difference was not significant statistically (women: 66.8\% vs. men: 61.7\%; AOR = 1.21;
Table 1. Baseline characteristics of people with type 2 diabetes $(n=889)$.

\begin{tabular}{lcc}
\hline Characteristics & Number & Percent \\
\hline Age & 388 & 43.6 \\
$\quad$ Up to 50 years & 501 & 56.4 \\
$\quad>50$ years & & \\
Marital status & 782 & 88.0 \\
$\quad$ Living with spouse & 107 & 12.0 \\
$\quad$ Not living with spouse & & \\
Education & 257 & 55.6 \\
$\quad$ Up to 5 years of schooling & 258 & 60.4 \\
$\quad>5$ years of schooling & & \\
Duration of disease & 360 & 40.5 \\
Up to 5 years & 529 & 59.5 \\
$\quad>5$ years & & \\
Physically active & 308 & 34.6 \\
Yes & 581 & 65.4 \\
No & &
\end{tabular}

Table 2. Association of metabolic syndrome and its individual components with Ischemic Heart Disease among people with type 2 diabetes.

\begin{tabular}{|c|c|c|c|c|}
\hline Factors & $\begin{array}{c}\text { Overall } \\
n(\%)\end{array}$ & $\begin{array}{l}\text { Having IHD } \\
(28 \% \text { of } n)\end{array}$ & $\beta$-value & $\begin{array}{c}\text { Odds Ratio } \\
\text { (95\% CI) }\end{array}$ \\
\hline Metabolic Syndrome & $\begin{array}{c}664 \\
(70.0)\end{array}$ & 85.1 & 1.17 & $\begin{array}{c}3.21 \\
(2.22-4.65)\end{array}$ \\
\hline -Hypertension & $\begin{array}{c}616 \\
(65.0)\end{array}$ & 70.3 & 0.33 & $\begin{array}{c}1.39 \\
(1.10-1.90)\end{array}$ \\
\hline -Microalbuminuria & $\begin{array}{c}312 \\
(32.9)\end{array}$ & 42.8 & 0.60 & $\begin{array}{c}1.83 \\
(1.36-2.45)\end{array}$ \\
\hline -Dyslipidemia & $\begin{array}{c}454 \\
(47.9)\end{array}$ & 58.7 & 0.61 & $\begin{array}{c}1.84 \\
(1.38-2.45)\end{array}$ \\
\hline -Obesity & $\begin{array}{c}400 \\
(42.2)\end{array}$ & 58.7 & 0.94 & $\begin{array}{c}2.57 \\
(1.91-3.43)\end{array}$ \\
\hline
\end{tabular}

$95 \% \mathrm{CI}=0.88-1.66)$

\section{DISCUSSION}

Summary of Key Findings: The unfolding global pandemic of diabetes has been shown to have its greatest adverse impact in the low and middle income countries as they account for nearly $80 \%$ of diabetes related mortality across the world [21]. It is well established that among persons with type 2 diabetes, one of the leading causes of morbidity and mortality is the increased frequency of occurrence of cardiovascular disease [3,4]. In our study, nearly one third of people with type 2 diabetes had an established diagnosis of IHD. This is somewhat closer to around 25\% and 28\% prevalence observed in 
Table 3. Gender differences in Metabolic Syndrome and its individual components among people with type 2 diabetes.

\begin{tabular}{lcccccc}
\hline \multicolumn{1}{c}{$\begin{array}{c}\text { Metabolic Syndrome and } \\
\text { Components }\end{array}$} & \multicolumn{2}{c}{ Proportions (\%) } & \multicolumn{2}{c}{ Unadjusted } & \multicolumn{2}{c}{ Adjusted } \\
\hline & Men (42.5) & $\begin{array}{c}\text { Women } \\
\mathbf{( 5 7 . 5 )}\end{array}$ & $\boldsymbol{\beta}$-value & $\begin{array}{c}\text { Odds Ratio } \\
\mathbf{( 9 5 \% ~ C I )}\end{array}$ & $\begin{array}{c}\boldsymbol{\beta} \text {-value } \\
\text { Odds Ratio } \\
\mathbf{( 9 5 \% ~ C I ) ~}\end{array}$ \\
\hline Metabolic Syndrome & 63.5 & 73.8 & 0.48 & $1.62(1.22-2.15)$ & 0.49 & $1.63(1.18-2.24)$ \\
-Hypertension & 61.7 & 66.8 & 0.22 & $1.25(1.00-1.65)$ & 0.19 & $1.21(0.88-1.66)$ \\
-Microalbuminuria & 39.7 & 29.0 & -0.48 & $0.62(0.47-0.82)$ & -0.37 & $0.70(0.51-0.95)$ \\
-Dyslipidemia & 56.8 & 42.8 & -0.57 & $0.57(0.44-0.74)$ & -0.55 & $0.58(0.43-0.78)$ \\
-Obesity & 21.4 & 54.1 & 1.46 & $4.31(3.18-5.84)$ & 1.54 & $4.70(3.30-6.70)$ \\
\hline
\end{tabular}

Adjusted odds ratios were calculated after adjusting for age, physical activity and smoking status.

South India and Iran respectively [22,23]. However, the prevalence found in our study is much higher than 13 and $10 \%$ seen in developed countries [24,25], consolidating the finding of increased burden of the problem in this part of the world. We believe that this may once again be pointing to the well established notion of "Asian Indian Phenotype" associated with increased insulin resistance, higher abdominal adiposity etc, along with decreased physical activity and dietary patterns that put Asian Indians more at risk of diabetes and premature coronary artery disease [26] when compared to other ethnicities.

The clustering of risk factors for CVD is seen as a universal phenomenon among people with type 2 diabetes [10-14]. MS is also a stronger and independent predictor for heart disease in type 2 diabetic patients [10, 14]. Like previous studies that consistently report over two-thirds of the diabetic patients to have this risk factor clustering $[10,13]$, we revealed that nearly three-fourths of our study participants also had MS. Additionally, it was observed that IHD was more likely among subjects with MS as compared to those without MS. Different studies show varied results regarding the role of individual components of metabolic syndrome in predicting the presence and severity of CVD. Fasting blood glucose, hypertension, obesity and dyslipidemia have all been found in different studies to be more significantly associated with CVD relative to one another $[13,27,28]$, however, some researchers identify central obesity to be the strongest predictor among all [29]. In our study also, the component with the strongest association with IHD was obesity.

We also analyzed in our study, the gender differences in the prevalence of MS and its components. A higher prevalence of clustering of IHD risk factors is seen among women, both diabetic and non-diabetic, in developing countries. $[12,13,18]$. This is in contrast to what is observed among general population in developed countries like US, where the prevalence of MS is higher among men [30]. However, we endorse the previous re- sults from developing countries that MS and its risk factor clustering are higher in women with type 2 diabetes compared to men. In a recently published communitybased study, it is reported that women in developing countries have higher clustering of behavioral risk factors than men [19], which may account for their being more at risk for MS. Of the various MS components, obesity, the factor identified with the strongest association with IHD as discussed earlier, was substantially higher among women in our study, after adjusting for the confounders. One of the possible explanations for this high prevalence of obesity among women with type 2 diabetes could be the socio-cultural restrictions that limit women's outdoor activities both work related and recreational like exercise and walking, rendering them more physically inactive than men.

Limitations: In this study limitations should be considered before interpreting these results for all people with diabetes. Being a cross-sectional study, temporal relation cannot be assessed for ischemic heart disease with MS and its components. Since IHD in diabetics is often silent with respect to symptoms, non-invasive studies such as myocardial scintigraphy and dobutamine stress echocardiography should have been performed for a thorough evaluation of the cardiac status. However, these investigations being costly were not used to screen patients for IHD, which may have resulted in under estimation of the results. We have no data about waist circumference, hence restricted only to WHO criteria of MS. Moreover, study participants belonged to urban part of country hence should be generalized for rural population with caution.

Nevertheless, results of this multi-centre study are sufficient enough to conclude that a large proportion of people with type 2 diabetes were suffering from MS and its individual components. Furthermore, each of the components was significantly associated with ischemic heart disease and the likelihood of IHD increased over three times when these risk factors were clustered according to the WHO criteria of MS. 


\section{CONCLUSION}

The results of this investigation provide useful information to formulate primary preventive strategies to prevent and delay the onset of serious avoidable complications of diabetes. In the light of the above findings, it is recommended that intervention programs promoting healthy life styles should be encouraged and made accessible to the common people. These may include accessibility to healthy food, provision of walking tracks and playgrounds that are safe, clean and socially acceptable particularly for the women, and promoting health education on the prevention of complication of diabetes among patients. In addition, more research is required to obtain information regarding the disease as it behaves in our population.

\section{ACKNOWLEDGEMENTS}

We are grateful to the management staff of all the study clinics for their permission to conduct the study and their help throughout the study period. We are very much indebted to all patients who agreed to participate in the study. We also acknowledge Mr. Iqbal Azam (Assistant Professor-Biostatistics) and Dr. Saima Akhund (Senior Instructor-Research) Aga Khan University, Karachi for reviewing manuscript.

\section{REFERENCES}

[1] Shaw, J.E., Sicree, R.A. and Zimmet, P.Z. (2010) Global estimates of the prevalence of diabetes for 2010 and 2030. Diabetes Research and Clinical Practice, 87, 4-14. doi:10.1016/j.diabres.2009.10.007

[2] Shera, A.S., Basit, A., Fawwad, A., Hakeem, R., Ahmedani, M.Y., Hydrie, M.Z. and Khwaja, I.A. (2010) Pakistan National Diabetes Survey: Prevalence of glucose intolerance and associated factors in the Punjab Province of Pakistan. Primary Care Diabetes, 4, 79-83. doi:10.1016/j.pcd.2010.01.003

[3] Khuwaja, A.K., Rafique, G., Azam, I. and White, F. (2004) Macrovascular complications and their associated factors among persons with type 2 diabetes in Karachi, Pakistan-A multi-centre study. Journal of Pakistan Medical Association, 54, 60-66.

[4] Coccheri, S. (2007) Approaches to prevention of cardiovascular complications and events in diabetes mellitus. Drugs, 67, 997-1026. doi:10.2165/00003495-200767070-00005

[5] Hoerger, T.J. and Ahmann, A.J. (2008) The impact of diabetes and associated cardiometabolic risk factors on members: Strategies for optimizing outcomes. Journal of Managed Care Pharmacy, 14, 2-14.

[6] Khuwaja, A.K., Khowaja, L.A. and Cosgrove, P. (2010) The economic costs of diabetes in developing countries: Some concerns and recommendations. Diabetologia, 53, 89-90. doi:10.1007/s00125-009-1581-7

[7] Roaeid, R.B. and Kablan, A.A. (2010) Diabetes mortality and causes of death in Benghazi: A 5-year retrospective analysis of death certificates. Eastern Mediterranean Health Journal, 16, 65-69.

[8] American Diabetes Association (ADA) (2009) Standards of medical care in diabetes 2009. Diabetes Care, 32, S13-S61. doi:10.2337/dc09-S013

[9] Khuwaja, A.K. (2005) Microalbuminuria among patients with type 2 diabetes mellitus: Early screening and intervention required. Hong Kong Medical Journal, 11, 70.

[10] Tong, P.C., Kong, A.P., So, W.Y., Yang, X., Ho, C.S., Ma, R.C., Ozaki, R., Chow, C.C., Lam, C.W., Chan, J.C. and Cockram, C.S. (2007) The usefulness of the International Diabetes Federation and the National Cholesterol Education Program's Adult 10 Treatment Panel III definitions of the metabolic syndrome in predicting coronary heart disease in subjects with type 2 diabetes. Diabetes Care, 30, 1206-1211. doi:10.2337/dc06-1484

[11] Koehler, C., Ott, P., Benke, I. and Hanefeld, M. (2007) Comparison of the prevalence of the metabolic syndrome by WHO, AHA/NHLBI, and IDF definitions in a German population with type 2 diabetes: The Diabetes in Germany (DIG) Study. Hormone and Metabolic Research, 39, 632-635. doi:10.1055/s-2007-985816

[12] Ahmed, S., Ahmed, S.A. and Ali, N. (2010) Frequency of metabolic syndrome in type 2 diabetes and its relationship with insulin resistance. Journal of Ayub Medical College, 22, 22-27.

[13] Ahmed, N., Ahmad, T., Hussain, S.J. and Javed, M. (2010) Frequency of metabolic syndrome in patients with type-2 diabetes. Journal of Ayub Medical College, 22, 139-142.

[14] de Simone, G., Devereux, R.B., Chinali, M., Best, L.G., Lee, E.T., Galloway, J.M. and Resnick, H.E, Strong Heart Study Investigators (2007) Prognostic impact of metabolic syndrome by different definitions in a population with high prevalence of obesity and diabetes: The strong heart study. Diabetes Care, 30, 1851-1856. doi:10.2337/dc06-2152

[15] Song, S.H. and Hardisty, C.A. (2008) Diagnosing metabolic syndrome in type 2 diabetes: Does it matter? An International Journal of Medicine, 101, 487-491. doi:10.1093/qjmed/hcn034

[16] Jafar, T.H., Qadri, Z. and Chaturvedi, N. (2008) Coronary artery disease epidemic in Pakistan: More electrocardiographic evidence of ischaemia in women than in men. Heart, 94, 408-413. doi:10.1136/hrt.2007.120774

[17] Jafar, T.H. (2006) Women in Pakistan have a greater burden of clinical cardiovascular risk factors than men. International Journal of Cardiology, 106, 348-354.

[18] Njelekela, M.A., Mpembeni, R., Muhihi, A., Mligiliche, N.L., Spiegelman, D., Hertzmark, E., Liu, E., Finkelstein, J.L., Fawzi, W.W., Willett, W.C. and Mtabaji, J. (2009) Gender-related differences in the prevalence of cardiovascular disease risk factors and their correlates in urban Tanzania. BMC Cardiovascular Disorders, 17, 30. doi:10.1186/1471-2261-9-30

[19] Khuwaja, A.K. and Kadir, M.M. (2010) Gender differences and clustering pattern of behavioural risk factors for chronic noncommunicable diseases: Community-based 
study from a developing country. Chronic Illness, 6, 163170. doi:10.1177/1742395309352255

[20] World Health Organization (WHO) Definition, diagnosis and classification of diabetes mellitus and its complications. Part 1: Diagnosis and classification of diabetes mellitus. WHO, Geneva, 1999.

[21] World Health Organization (2011) Media Centre: Diabetes. http://www.who.int/mediacentre/factsheets/fs312/en/

[22] Mohan, V., Deepa, R., Rani, S.S. and Premalatha, G. (2001) Prevalence of coronary artery disease and its relationship to lipids in a selected population in South India: The Chennai Urban Population Study (CUPS No. 5). Journal of American College of Cardiology, 38, 682-687. doi:10.1016/S0735-1097(01)01415-2

[23] Janghorbani, M., Amini, M. and Tavassoli, A. (2006) Coronary heart disease in type 2 diabetes mellitus in Isfahan, Iran: Prevalence and risk factors. Acta Cardiologica, 61, 13-20. doi:10.2143/AC.61.1.2005135

[24] Harris, S.B., Naqshbandi, M., Bhattacharyya, O., Hanley, A.J., Esler, J.G. and Zinman, B. (2011) Major gaps in diabetes clinical care among Canada’s First Nations: Results of the CIRCLE study. Diabetes Research and Clinical Practice, 92, 272-279. doi:10.1016/j.diabres.2011.02.006

[25] DAI Study Group (2004) The prevalence of coronary heart disease in type 2 diabetic patients in Italy: The DAI study. Diabetic Medicine, 21, 738-745. doi:10.1111/j.1464-5491.2004.01230.x
[26] Deepa, R., Sandeep, S. and Mohan, V. (2006) Abdominal obesity, visceral fat and type 2 diabetes-Asian Indian phenotype. In: Mohan, V. and Rao, G.H.R., Eds., Type 2 diabetes in South Asians: Epidemiology, Risk Factors and Prevention. Jaypee Brothers Medical Publishers Ltd., New Delhi, 138-152.

[27] Hwang, I.C., Kim, K.K., Jee, S.H. and Kang, H.C. (2011) A comparison of predictability of cardiovascular events between each metabolic component in patients with metabolic syndrome based on the revised national cholesterol education program criteria. Yonsei Medical Journal, 52, 220-226. doi:10.3349/ymj.2011.52.2.220

[28] Bosevski, M., Pemovska, G., Bosevska, G. and Georgievska-Ismail, L. (2010) Clinical role of estimation metabolic syndrome's components in type 2 diabetic population with symptomatic coronary artery disease-A comparison of two criteria. Medicinski Arhiv, 64, 144-146.

[29] Doi, Y., Ninomiya, T., Hata, J., Yonemoto, K., Arima, H., Kubo, M., Tanizaki, Y., Iwase, M., Iida, M. and Kiyohara, Y. (2009) Proposed criteria for metabolic syndrome in Japanese based on prospective evidence: The Hisayama study. Stroke, 40, 1187-1194. doi:10.1161/STROKEAHA.108.531319

[30] Ford, E.S., Li, C. and Zhao, G. (2010) Prevalence and correlates of metabolic syndrome based on a harmonious definition among adults in the US. Journal of Diabetes, 2, 180-193. doi:10.1161/STROKEAHA.108.531319 